\title{
Quantitative Analysis of a Steel Billet Surface Flaw Detection System by Means of a Finite Element Method
}

\author{
Sungwoo Bae* and Hongyeob Lee ${ }^{\dagger}$
}

\begin{abstract}
The surface inspection of a steel billet is a common practice in the steel manufacturing process prior to hot rolling to produce steel wire for tire cord. This billet surface inspection is an important process because flaws on the surface may cause major failures during the product manufacturing phase. This paper presents a computer simulation based on a finite element method for a magnetic flaw detector with a function of the current intensity, the number of coil turns, and the billet proceeding speed during the production phase based on the typical condition of conventional apparatus. Based on the simulation result, the magnitude of the electromagnetic field on the surface diminished with distance from the electromagnet. In addition, the increased current intensity and the increased number of coil turns actually induced a stronger electromagnetic field on the billet surface. On the other hand, the proceeding speed of a billet in its production line had no significant effects. The result in this study may assist to reduce trial and error and to minimize the opportunity costs during the optimization process by applying the findings of this study into the operation condition in the steel billet production line.
\end{abstract}

Keywords: Electromagnet, Surface flaw detection, Steel billet, Magnetic field

\section{Introduction}

This paper presents a computer simulation by a finite element method for a billet surface flaw detection system that uses a magnetic field to discover a defective product. A steel billet is a semi-finished casting product which will be manufactured directly by continuous billet casting machine or indirectly by a hot-rolling process [1]. After the processes of hot-rolling and drawing billet, this billet becomes a final product such as wire or bar stock [2]. Steel manufacturers commonly use surface flaw detection apparatus prior to a hot-rolling process to detect defects on the surface of a steel billet in order to prevent a product lifecycle reduction and possible failures such as breakages during the drawing process and fracture in the final product [3-5]. Therefore, full-number inspection with a flaw detection system is a recent trend in the steel manufacturing industry in order to reduce the cost of poor quality. Particularly, a non-contact type flaw detection system is preferred because it can be mounted on-line prior to a rolling process [6].

Steel manufacturers widely use magnetic type flaw detection apparatus or a magnetic particle inspector that generates a magnetic field on the billet surface. A magnetic field source is located outside of the billet surface and magnetizes a base metal to identify a defective product. This billet surface magnetization allows fluorescent

$\dagger$ Corresponding Author: Dept. of Electrical Engineering, Yeungnam University, Korea. (tony0217@naver.com)

* Dept. of Electrical Engineering, Yeungnam University, Korea (sbae@yu.ac.kr)

Received: March 4, 2016; Accepted: May 25, 2016 magnet particles to be concentrated on the flaw surfaces such as crack, holes, and scratches [7, 8]. Steel manufacturers usually change the operation conditions of this flaw detection process to enhance its efficiency and productivity. Otherwise, the efficiency of flaw detection may vary, and the possibility of major failures such as crack and breakage can be increased [7]. A conventional approach to improve and stabilize this surface flaw detection efficiency was based on an empirical method. This empirical method requires changing operation conditions such as billet production speed, output current of an electromagnet, and gap between a magnetic source and an object to be inspected. These operational conditions change in the billet surface flaw detection system may affect its flaw detection efficiency. In addition, it usually takes long time to stabilize a billet surface flaw detection process based on this empirical method with trial and error.

On the other hand, this paper proposes a computer simulation using a finite element method to provide quantitative understandings of a magnetized surface and to suggest a better approach for the optimization of an operational condition design. For the computer simulation, this study used a 3D finite element method simulation analysis. Thus, its analysis results would assist to understand the phenomena of the magnetized flaw detection system so that this computer simulation study can contribute not only to minimize the opportunity costs and time, but also to enhance the billet surface flaw detection efficiency. The scope of simulation in this study is limited to observe the electromagnetic field changes on the one surface of a moving billet with a function of moving speed, 
current intensity, and turns of electromagnets. Other conditions such as a defector size, a defector shape on the billet surface and dimensional changes in the material and electromagnets are out of scope in this paper. The magnetic flux density induced by different speed and different electromagnetic condition was investigated and analyzed. The simulated results can be used to assist in the understanding of the behavior of a magnetic field on the billet surface.

The organization of the remainder in this paper is as follows: Section 2 discusses the dimensional information and simulation conditions of a magnetic flaw detection system based on the typical operation condition of a steel manufacturing factory. Section 3 describes the various simulation results of the magnetic flux density on the billet surface based on different current intensities, different number of coil turns, and different billet proceeding speed in its surface flaw detection device. This paper concludes in Section 4 with the summary of findings.

\section{Finite Element Method Simulation}

This section describes the dimensional information and operational conditions of magnetic flaw detection apparatus investigated in the finite element method (FEM) simulation in this paper. In order to simulate a magnetic flaw detector in the similar condition to a practical process, this study used real process data and information based on the practical apparatus in a steel manufacturing factory in Korea. The cross-sectional area of a simulated steel billet which had a square shape was between $144 \mathrm{~cm}^{2}$ (i.e., 120 $m m \times 120 \mathrm{~mm}$ ) and $256 \mathrm{~cm}^{2}$ (i.e., $160 \mathrm{~mm} \times 160 \mathrm{~mm}$ ). The nominal length of a billet varied from $3,600 \mathrm{~mm}$ to 12,000 $\mathrm{mm}$ depends on the manufacturers. The investigated practical operational conditions are shown in Table 1.

\subsection{FEM simulation design and layout}

The simulation study in this paper was carried out with two electromagnets mounted vertically as shown in Fig. 1. A steel billet of which dimensional size was $1,000 \mathrm{~mm}(L)$ $\times 160 \mathrm{~mm}(W) \times 160 \mathrm{~mm}(H)$ was designed based on the actual dimension of a practical billet to analyze an electromagnetic effect as depicted in Fig. 1. Although the investigated practical length of a billet was $10,200 \mathrm{~mm}$, the simulated length of a billet was assumed to be $1,000 \mathrm{~mm}$

Table 1. Practical operational conditions.

\begin{tabular}{c|c}
\hline Material & Steel \\
\hline Speed of a Billet & $0.7 \mathrm{~m} / \mathrm{s}(42 \mathrm{MPM})$ \\
\hline Practical Billet Dimension & $10,200 \mathrm{~mm}(\mathrm{~L}) \times 160 \mathrm{~mm}(\mathrm{~W}) \times 160 \mathrm{~mm}(\mathrm{H})$ \\
\hline Simulated Billet Dimension & $1,000 \mathrm{~mm}(\mathrm{~L}) \times 160 \mathrm{~mm}(\mathrm{~W}) \times 160 \mathrm{~mm}(\mathrm{H})$ \\
\hline Coil/Core Diameter & $40 \mathrm{~mm} / 40-20 \mathrm{~mm}$ \\
\hline Coil Specification & $10,000 \mathrm{Turns}, 10 \mathrm{~A}$ \\
\hline Distance between Coil Billets & $60 \mathrm{~mm}$ \\
\hline
\end{tabular}

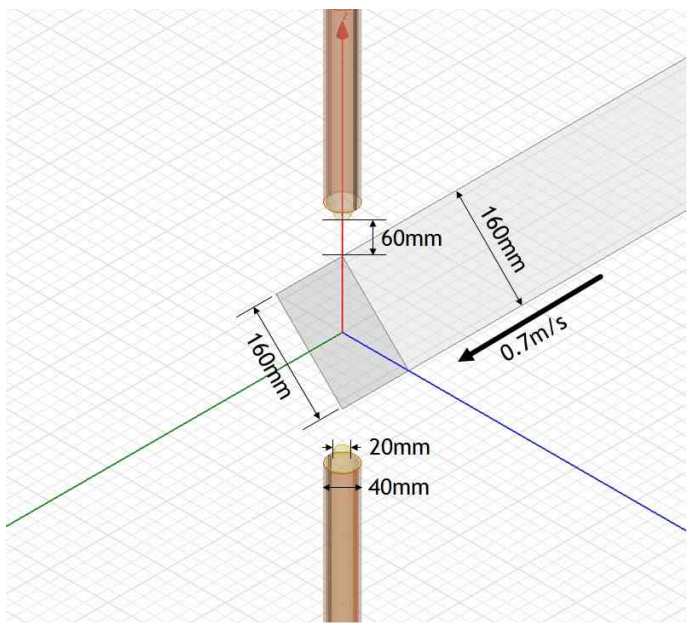

(a)

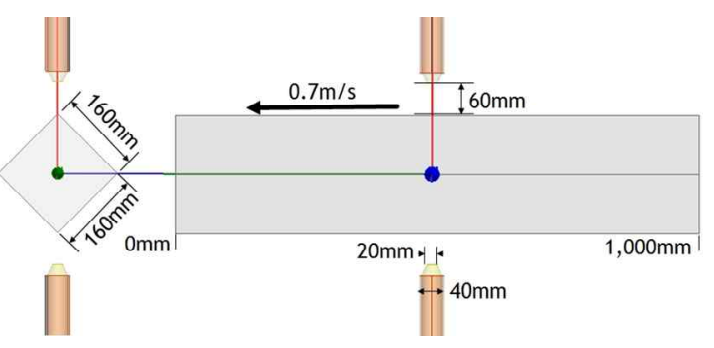

(b)

Fig. 1. Dimensional information of the investigated magnetic billet surface flaw detector. (a) 3D layout dimension. (b) 2D layout dimension.

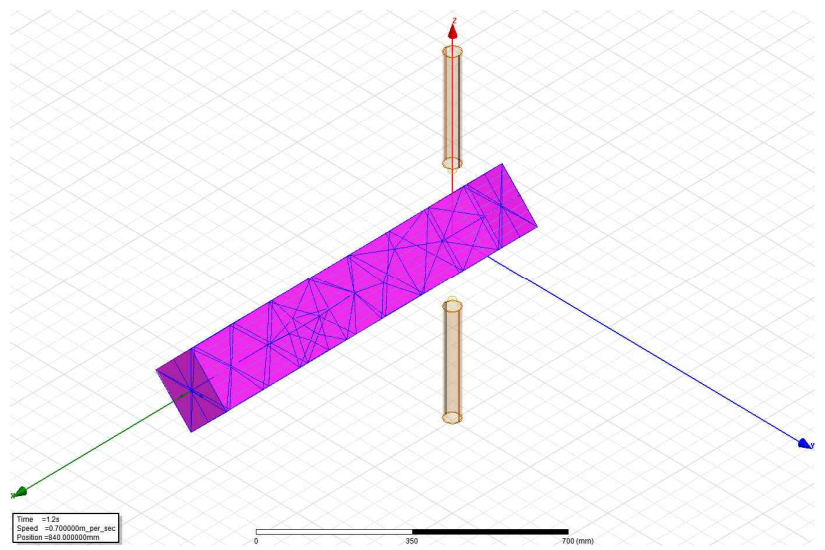

Fig. 2. Refined meshes of the investigated simulation study.

$(L)$ to reduce the analysis time of the simulation study in this paper as described in Table 1. Refined meshes in the steel billet are shown in Fig. 2. Steel was selected for a material property because the chemical composition of the alloy produced by manufacturers can be changed. A magnetic transient mode was selected in the finite element method simulation software to conduct the simulation study. 


\subsection{Simulation conditions with practical process data}

In the practical detector of a steel manufacturing factory, four electromagnets are usually mounted at each corner of a billet to induce the magnetic field on the billet surface. In this simulation study, however, it was assumed that two electromagnets were installed at the top and bottom side of the apparatus as shown in Fig. 1 in order to clarify the effect of the specific coil. In other words, this simplification was required to eliminate the cross-talk between electromagnets. The area of interest was also limited to the surface of the first quadrant because the billet surface flaw detector has a symmetric structure.

The purpose of this paper is to provide quantitative explanations for those who want to change the operation conditions. A practical operational condition in Table 1 was considered as the basic condition (i.e., condition I) for comparing with other simulation conditions in Table 2. Electromagnetic field distribution on the billet surface was investigated with this basic condition. The finite element method simulation result with this condition $I$ is shown in Fig. 3. In the simulation condition I in Table 2, 9 inspection points (i.e., $0 \mathrm{~mm}, 20 \mathrm{~mm}, 40 \mathrm{~mm}, 60 \mathrm{~mm}, 80 \mathrm{~mm}, 100 \mathrm{~mm}$, $120 \mathrm{~mm}, 140 \mathrm{~mm}$, and $160 \mathrm{~mm}$ ) on the widthwise direction of the surface were investigated to analyze the distribution

Table 2. Simulation Conditions.

\begin{tabular}{c|c|c|c|c}
\hline Cond. & Speed & Current & Turns & $\begin{array}{c}\text { Widthwise } \\
\text { Inspection Points }\end{array}$ \\
\hline I & $0.7 \mathrm{~m} / \mathrm{s}$ & $10 \mathrm{~A}$ & 10,000 & $\begin{array}{c}0 \mathrm{~mm}, 20 \mathrm{~mm}, 40 \mathrm{~mm}, \\
60 \mathrm{~mm}, 80 \mathrm{~mm}, \\
100 \mathrm{~mm}, 120 \mathrm{~mm}, \\
140 \mathrm{~mm}, 160 \mathrm{~mm}\end{array}$ \\
\hline II & $0.7 \mathrm{~m} / \mathrm{s}$ & $\begin{array}{c}5 \mathrm{~A} \\
10 \mathrm{~A}\end{array}$ & 10,000 & $\begin{array}{c}80 \mathrm{~mm} \\
(\text { Center })\end{array}$ \\
\hline III & $0.7 \mathrm{~m} / \mathrm{s}$ & $10 \mathrm{~A}$ & $\begin{array}{c}10,000 \\
20,000\end{array}$ & $\begin{array}{c}80 \mathrm{~mm} \\
(\text { Center })\end{array}$ \\
\hline IV & $0.35 \mathrm{~m} / \mathrm{s}$ & $10 \mathrm{~A}$ & 10,000 & $\begin{array}{c}80 \mathrm{~mm} \\
(\text { Center })\end{array}$ \\
\hline
\end{tabular}

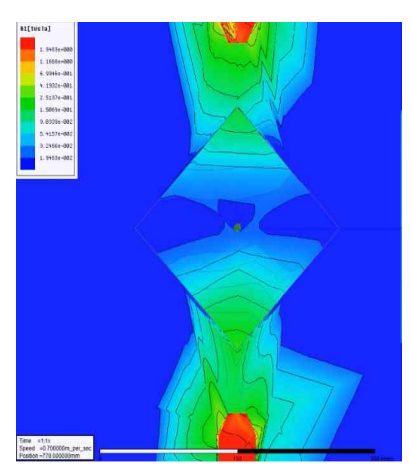

(a)

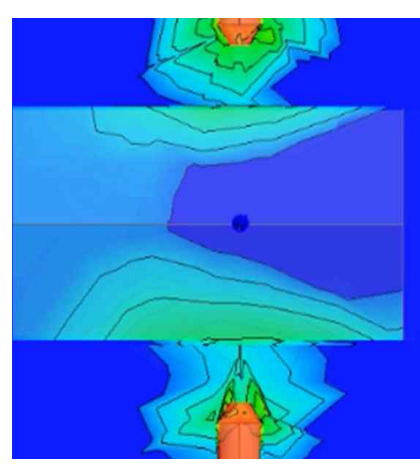

(b)
Fig. 3. Finite element method simulation result with the simulation condition I: (a) Front view of the result; (b) Side view of the result. and the degradation of the magnetic field in order to provide a quantitative understanding of a magnetized billet surface. Other simulation conditions in Table 2 were only investigated at the $80 \mathrm{~mm}$ point in the widthwise direction to compare the effect of the different conditions because unconsidered two electromagnets on the parallel direction which can be mounted to magnetize the parallel edge in the practical process can magnetize the billet surface from the widthwise center to edge position of the billet. The simulation conditions II and III were designed to observe the magnetic flux density changes at the center point of the billet width (i.e., $80 \mathrm{~mm}$ ) as a function of current intensities and coil turns. Lastly, a billet proceeding speed in the production line was used as a parameter to obtain the surface flaw detection effect by a billet production speed.

\section{Results and Discussions}

This section describes the simulation results of the magnetic particle inspector that were obtained with the simulation conditions described in Table 2.

\subsection{Condition I - Electromagnetic field distribution}

Fig. 3 shows the finite element method simulation result

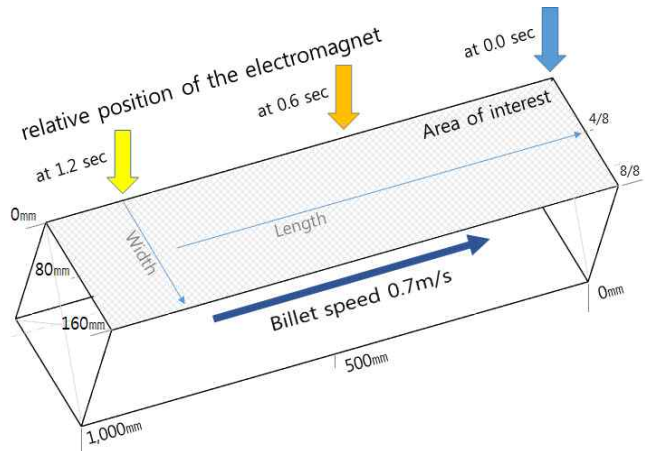

(a)

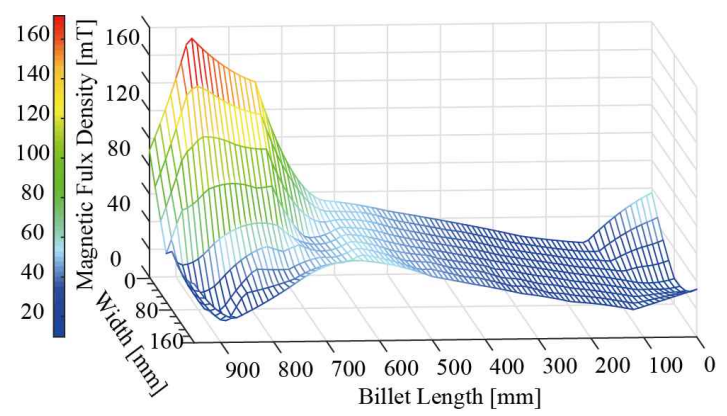

(b)

Fig. 4. Magnetic field distribution on the billet surface at $1.2 \mathrm{sec}$ with the simulation condition I: (a) Relative position of the electromaget with the simulated billet; (b) Magnetic flux density according to the width and billet length. 
with the condition I in Table 1. Fig. 4(a) depicts the relative position of the electromagnet with the simulated billet. Fig. 4(b) illustrates the magnetic field distribution on the billet surface at $1.2 \mathrm{sec}$ with the simulation condition I in Table 1. As depicted in Fig. 4(b), the magnetic flux density of the electromagnetic field on the surface diminished as it is apart from the center of the electromagnet. This magnetic flux density on the billet shows the maximum value beneath the electromagnet and the minimum value at the diagonal edge. The vertical deviation of the electromagnetic field was homogenized as the magnetized area departed from the electromagnetic source, and the magnetic flux density started to dissipate while the billet proceeded forward.

\subsection{Condition II - Effect of current intensity}

Fig. 5 shows the magnetic flux density by billet width with different current intensities which were the simulation condition II in Table 2. Similarly, Fig. 6 depicts the magnetic flux density by billet length with these different current intensities. As shown in Figs. 5 and 6, the increasing current intensity from $5 \mathrm{~A}$ to $20 \mathrm{~A}$ leads to a gradual increase in the magnetic field density along the

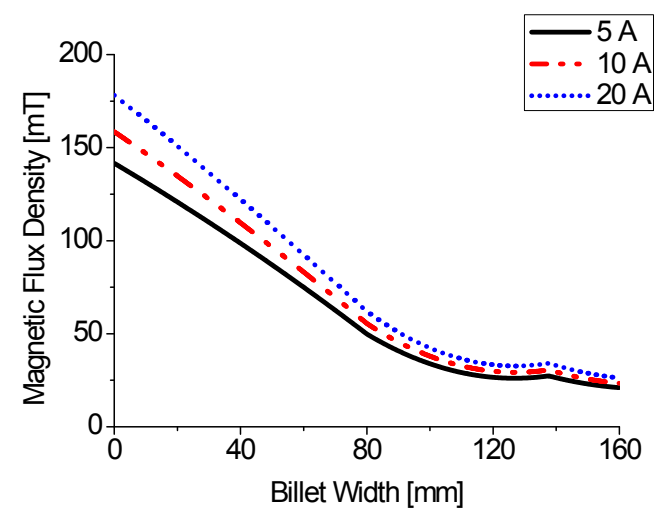

Fig. 5. Magnetic flux density by width with different current intensities.

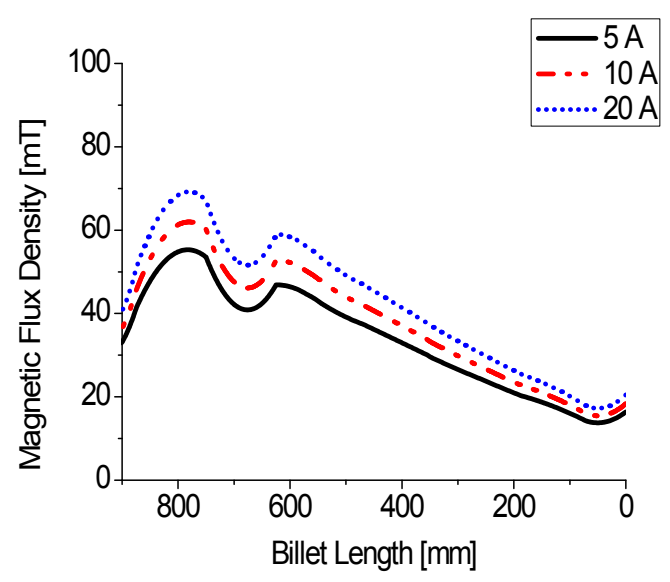

Fig. 6. Magnetic flux density by length with different current intensities. entire width and length of the billet surface. The simulation result with the 20 A condition showed the maximum magnetic flux density among the other conditions, and the magnetic flux density diminished with distance. As illustrated in Fig. 6, in the lengthwise, the magnetic flux density which was obtained at the widthwise center of the billet surface showed the maximum value beneath the electromagnet and diminished gradually as the electromagnet departed.

\subsection{Condition III - Number of coil turns effect}

Fig. 7 shows the magnetic flux density by billet width with different number of coil turns (i.e., 5,000 turns, 10,000 turns, and 20,000 turns) which were the simulation condition III in Table 2. In the same way, Fig. 8 depicts the magnetic flux density by billet length with these different number of coil turns. As shown in Figs. 7 and 8, the influence of the number of coil turn condition changes was similar to that of current intensity changes. The increase in the number of coil turns from 5,000 to 20,000 results in the magnetic flux density increase along the entire width and length of the billet surface.

\subsection{Condition IV - Effect of Billet Proceeding Speeds}

Fig. 9 shows the magnetic flux density by billet width

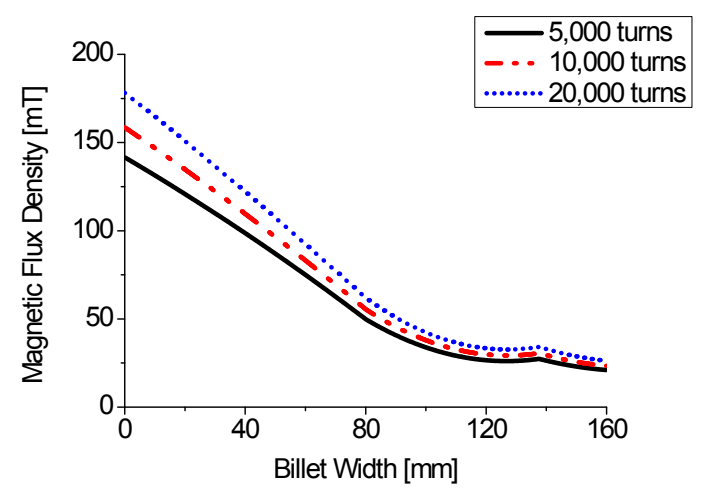

Fig. 7. Magnetic flux density by width with different coil turns.

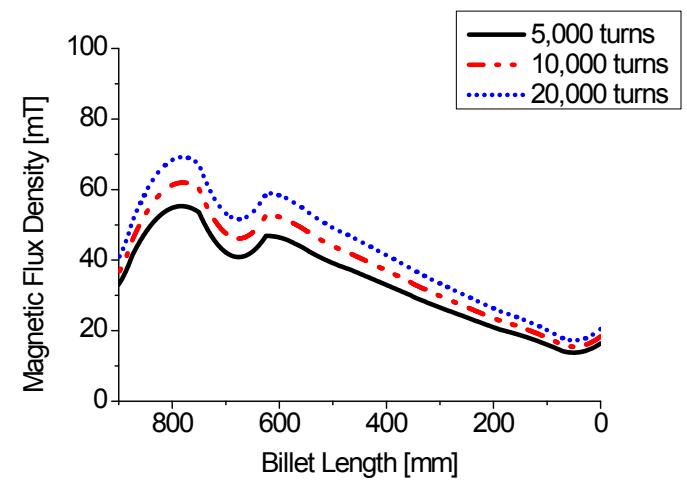

Fig. 8. Magnetic flux density by length with different coil turns. 


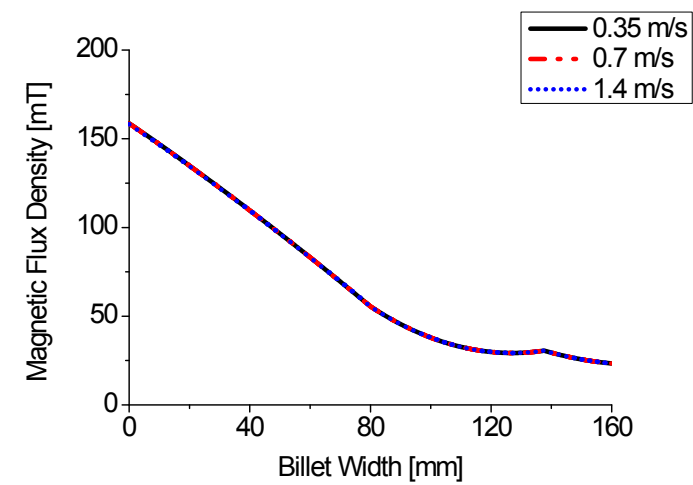

Fig. 9. Magnetic flux density by width with different billet speeds.

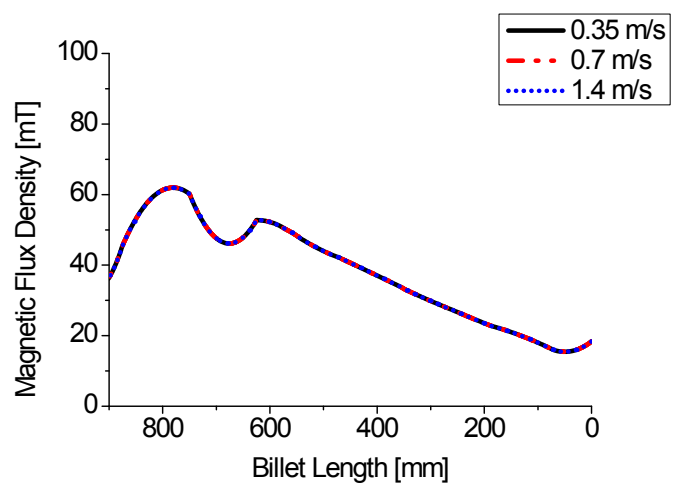

Fig. 10. Magnetic flux density by length with different billet speeds.

with different billet proceeding speeds (i.e., $0.35 \mathrm{~m} / \mathrm{s}, 0.7$ $\mathrm{m} / \mathrm{s}$, and $1.4 \mathrm{~m} / \mathrm{s}$ ) which were the simulation condition IV in Table 2. Likewise, Fig. 10 illustrates the magnetic flux density by billet length with these different billet proceeding speeds. As shown in Figs. 9 and 10, there were not any significant differences in the simulation results with different billet proceeding speeds.

In summary, these simulation results in this section can explain the following magnetic field effect on the billet surface. The magnetic field distribution created by the electromagnet became homogenized gradually, and it dissipated with distance as shown in Fig. 4. As shown in Figs. 5 8, the magnetic field density gradually increased as the current intensity and the number of coil turns rose. On the other hand, the speed of a still billet production line did not produce a significant change to the magnetic field distribution. Only the condition changes of the electromagnet induced different phenomena on the billet surface.

\section{Conclusions}

This paper proposed a computer simulation using a finite element method to analyze the electromagnet field changes on the steel billet surface. The simulation layouts based on a practical magnetic particle inspector for a billet in the steel manufacturing factory in Korea were designed to observe the difference from the standard operation condition. These simulation results may provide a better quantitative understanding on a magnetized billet surface with various conditions including changes in the current intensity, the number of coil turns, and the billet moving speed of its production line. Based on the simulation study in this paper, it can be concluded that the increased current intensity and the increased number of coil turns would actually induce a stronger electromagnetic field on the billet surface. On the other hand, the proceeding speed of a billet in its production line had no significant effects. Prior to change the actual operation condition in the steel billet production line, this computational modeling result would reduce trial and error and minimize the opportunity costs during the optimization process. The results in this paper can also be used to improve the detection efficiency of the billet surface flaw detection apparatus.

\section{Acknowledgements}

This research was supported by Basic Science Research Program through the National Research Foundation of Korea (NRF) funded by the Ministry of Science, ICT and Future Planning (NRF-2014R1A1A1036384).

\section{References}

[1] H. Sato, "The Iron and Steel Industry in Asia: Development and Restructuring," Institute of Developing Economies Discussion Paper, no. 210, pp. 1-35, Aug. 2009.

[2] W. Roberts, Hot rolling of steel, CRC Press, 1983.

[3] E. Fletcher, "MIDAS - High Speed Automatic Surface Inspection of Steel Billets," NDT International, vol. 12, no. 4, pp. 163-166, Aug. 1979.

[4] T. Nishimine, O. Tsuyama, T. Tanaka and H. Fujiwara, "Automatic magnetic particle testing system for square billets," in Conf. Rec. of the 1995 IEEE Industry Applications Conf., pp.1585-1590, vol. 2, 1995.

[5] H. Lee, Y. Lee, and S. Bae, "Electromagnetic Field Analysis on the Magnetic Surface Flaw Detection Apparatus," International Conference on Information and Convergence Technology for Smart Society, pp.1-2, Jan. 2016.

[6] W. A. Black, "Automatic inspection of steel billets," Non-Destructive Testing, vol. 4, no. 5, pp.323-329, Oct. 1971.

[7] M. J. Lovejoy, Magnetic particle inspection: a practical guide, Springer Science \& Business Media, 2012.

[8] J. Y. Lee, S. J. Lee, D. C. Jiles, M. Garton, R. Lopez 
and L. Brasche, "Sensitivity analysis of simulations for magnetic particle inspection using the finiteelement method," IEEE Transactions on Magnetics, vol. 39, no. 6, pp.3604-3606, Nov. 2003.

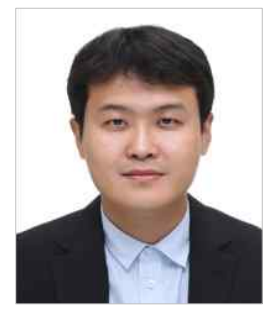

Sungwoo Bae received the B.S. degree from Hanyang University, Seoul, Korea, and the M.S.E. and Ph.D. degrees from the University of Texas at Austin, USA, all in electrical engineering, in 2006, 2009, and 2011, respectively. From 2012 to 2013, he was a senior research engineer with Power Center at Samsung Advanced Institute of Technology. He has been an Assistant Professor in the department of electrical engineering at Yeungnam University in Korea since 2013. In 2005, Dr. Bae was awarded the Grand Prize at the national electrical engineering design contest by the Minister of Commerce, Industry and Energy of the Republic of Korea.

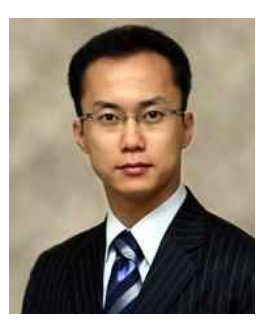

Hongyeob Lee received the Bachelor of electrical and computer engineering in 2005 from Hanyang University, Seoul, Korea, and the M.Sc. in the Material Science from Pohang University of Science and Technology in 2014. He is currently working toward the Ph.D. degree in the department of electrical engineering at Yeungnam University in Korea. 